http://dx.doi.org/10.18675/1981-8106.vol26.n51.p142-166

\title{
Educação de pessoas com implante coclear: uma análise de artigos internacionais e nacionais.
}

\author{
Education of people with cochlear implant: analysis of international and \\ national articles
}

Educación de personas con implantes cocleares: una revisión de los documentos internacionales y nacionales.

\author{
Regiane da Silva Barbosa' \\ Maria da Piedade Resende da Costa" \\ 'Universidade Federal de São Carlos (UFSCAR), São Paulo - Brasil. E-mail: \\ regiane.sbarbosa@gmail.com \\ "Universidade Federal de São Carlos (UFSCAR), São Paulo - Brasil. E-mail: piedade@ufscar.br
}

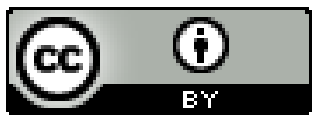

Educação: teoria e prática, Rio Claro, SP, Brasil - eISSN: 1981-8106

Está licenciada sob Licença Creative Common

\section{Resumo}

Este artigo discorre sobre as tendências internacionais e nacionais de pesquisas sobre educação de crianças com implante coclear (IC), procurando responder a seguinte questão: Quais as tendências da pesquisa internacional e nacional sobre Implante Coclear na área da educação? Teve como objetivo identificar, analisar e comparar pesquisas internacionais e nacionais sobre educação de pessoas com IC, uma vez que é crescente o número de crianças surdas em idade escolar que realizam a cirurgia. Método: pesquisa bibliográfica de caráter qualitativo e quantitativo, realizada por meio de busca de artigos publicados e indexados em Bancos de Dados no período de 2009 a 2013, utilizando-se o indexador cochlear implant implante coclear nos Bancos de Dados ERIC, SCIELO e LILACS respectivamente. Os resultados da pesquisa demonstram que há um déficit de pesquisas nacionais sobre o tema, enquanto pesquisas internacionais desenvolvem temas importantes para a educação, como alfabetização, leitura, inclusão e recursos importantes na educação de pessoa com IC. 
Conclusão: é preciso maior investimento em pesquisas nacionais na área, uma vez que há muitas lacunas e temas pouco ou nada explorados, como os temas encontrados em pesquisas internacionais que ficam como sugestão para futuras pesquisas.

Palavras-chave: Educação; Crianças com Implante Coclear; Pesquisas Internacionais; Pesquisas Nacionais.

\begin{abstract}
This article discourses on international and national research trends discussing children with cochlear implant's education, trying to answer the question: What are the trends in international and national research in education area on cochlear implants? Whose aim is to identify, analyze and compare national and international research on education of people with CI, since an increasing number of deaf children of school age who are submitted to surgery. Method: A literature study of qualitative and quantitative, performed by searching for published and indexed in Databases articles in the period 2009-2013, by using the cochlear implant indexer at the Bank of ERIC, LILACS and SciELO respectively. The research results demonstrated that there is a national deficit studies on the theme while international research develop important topics for education such as literacy, reading, inclusion and important resources in the education of people with CI. Conclusions: it's necessary more investment in national surveys in the area, since there are many gaps and underexplored or no themes, as the themes found in international research that are suggested for future researches.
\end{abstract}

Keywords: Education, Children with Cochlear Implants, International Research, National Research.

\title{
Resumen
}

Este artículo analiza las tendencias nacionales e internacionales en la investigación sobre la educación de los niños con Implantes Cocleares (IC), buscando responder a la siguiente pregunta: ¿Cuáles son las tendencias en la investigación nacional e internacional sobre IC en la educación? El objetivo es identificar, analizar y comparar la investigación nacional e internacional sobre la educación de las personas con IC, ya que es cada vez mayor el número de alumnos sordos que realizan la cirugía. Método: investigación bibliográfica cualitativa y cuantitativa, opinión, literatura, llevada a cabo a través de la búsqueda de artículos publicados e indexados en bases de datos de artículos en el período 2009-2013, utilizando el indexador Cochlear Implant / Implante Coclear en las bases de datos ERIC, SciELO y LILACS, respectivamente. Los resultados de la búsqueda muestran que hay una escasez nacional de investigación sobre el tema, mientras que la investigación internacional para desarrollar temas educativos importantes, como la alfabetización, la lectura, y la inclusión de 
características importantes en la educación de la persona con IC. Conclusión: necesitamos más inversiones en las encuestas nacionales ya que hay muchas lagunas y temas inexplorados como los que se encuentran en la investigación internacional que se sugieren para la investigación futura.

Palabras claves: Educación; Los niños con Implantes Cocleares; La Investigación Internacional; La Investigación Nacional.

\section{Introdução}

O Implante Coclear (IC) é um recurso tecnológico efetivo no tratamento da surdez praticado no Brasil há mais de 30 anos. Trata-se de um recurso tecnológico complexo, considerado por pesquisadores da área, como Hyppolito e Bento (2012), a prótese sensorial mais efetiva da medicina.

O IC estimula diretamente o nervo auditivo através de pequenos eletrodos que são colocados dentro da cóclea. É composto por duas unidades: externa e interna. A unidade externa é composta por processador de fala, antena e microfone que captam o som do ambiente, enviando-o ao processador de fala que os codifica em sinais elétricos que são enviados por radiofrequência para a unidade interna, a qual é constituída de um feixe de eletrodos conectados a um receptor que capta sinais elétricos. É indicado para pessoas surdas com surdez bilateral, severa e profunda que não se beneficiam com o uso de aparelhos auditivos conforme explicitam Costa Filho e Bevilacqua (1999).

Quanto antes for realizado o implante, maior serão os benefícios alcançados considerando-se ganho auditivo e desenvolvimento da linguagem, o que justifica a atual realização da cirurgia ainda no primeiro ano de vida das crianças com o referido tipo de surdez (MORET; BEVILACQUA; COSTA FILHO, 2007).

Não há consenso entre os pesquisadores sobre IC, em relação ao tempo necessário de uso do implante para que a pessoa desenvolva habilidades auditivas, mas é certo que o IC produz experiência de som e melhora o desempenho auditivo. No entanto, esse processo é longo, pois depende do estímulo recebido pelo paciente implantado após a ativação dos eletrodos (CAPOVILLA, 1998).

No Brasil, desde 1999 o Ministério da Saúde estabeleceu critérios de indicação para a realização do IC, determinando que em adultos a cirurgia deve ser realizada quando a pessoa com surdez neurossensorial severa ou profunda não se beneficia com uso de próteses auditivas, como o aparelho de amplificação sonora individual (AASI), e quando a mesma tenha condições psicológicas e motivação para uso do IC (BRASIL, 1999).

Além disso, a referida portaria estabeleceu que para realização do IC em pessoas com menos de 18 anos de idade, é preciso que ela, mesmo com uso do AASI, não consiga 
reconhecer palavras, e pertença a uma família que a apoie e motive no uso do recurso, além de ter em sua cidade condições adequadas de reabilitação. Os Centros/ núcleos cadastrados para realização do IC, além da cirurgia, devem oferecer serviços de:

(...) otorrinolaringologia, neurologia, genética clínica e pediatria; fonoaudiológicos: audiologia clínica incluindo adaptação de AASI e programas de reabilitação - terapias fonoaudiológicas distintas e adequadas a diferentes faixas etárias e necessidades de clientela; pedagógicos de acompanhamento em orientação escolar e serviços de audiologia educacional; serviço social; nutrição e enfermagem (...) (BRASIL, 1999, p.5).

Isto é, os centros/núcleos que realizam o IC devem oferecer atendimento e acompanhamento após a cirurgia, por uma equipe multidisciplinar, responsável não apenas pela ativação dos eletrodos implantados, mas também pelos ajustes necessários e por programas de estímulo e treinamento auditivo, pois "a medida que os canais mais próximos à abertura da cóclea são estimulados, tons cada vez mais agudos são ouvidos. (...) à medida que os canais mais profundos são estimulados, tons mais graves são ouvidos" (CAPOVILLA, 1998, p. 79).

Estes ajustes visam otimizar a recepção do som quando a pessoa está em uma conversa, daí a importância de acompanhamento pós cirurgia, por se tratar de um processo, no qual, aos poucos se melhora a recepção do som que deve ser reconhecido pela pessoa, pois em geral, estas pessoas não tiveram experiências auditivas prévias, a denominada memória auditiva, comum em crianças ouvintes e essencial ao desenvolvimento da linguagem oral. E mesmo para quem tem essa memória auditiva (pessoas com surdez pós lingual, com surdez adquirida após desenvolvimento da linguagem oral) o processo é complicado, pois os sons produzidos pelo IC são distorcidos (TANAMATI; COSTA; BEVILACQUA, 2011).

As primeiras experiências auditivas da pessoa com IC são confusas, com sons estranhos que precisam ser distinguidos e apreendidos pela pessoa implantada aos poucos. Como descreve Capovilla (1998), com o tempo o cérebro se acostuma com as sensações auditivas produzidas pelo IC, tornando-as confortáveis, naturais. Para isso é fundamental o programa de treinamento auditivo e educacional, de forma a encorajar a pessoa com IC a se comunicar pela audição e fala.

Além da falta de memória auditiva, da distorção dos sons, há muitos ruídos ambientais que comprometem a audição e distinção dos sons, em especial sons da fala. Daí a importância do acompanhamento multiprofissional pós implante, estabelecido pelo Ministério da Saúde, objetivando o sucesso da pessoa implantada.

Com o passar do tempo, os critérios para a indicação do IC para crianças com surdez se expandiram, proporcionando um aumento no número de pessoas com IC (TANAMATI; COSTA; BEVILACQUA, 2011). 
Os benefícios da pessoa com IC são particulares, uma vez que diferentes estudiosos da área, dentre eles Tanamati, Costa e Bevilacqua (2011, p. 366) pontuam que "a etiologia, a idade na implantação, a presença de audição residual, a reabilitação auditiva, a participação familiar no processo terapêutico, podem potencialmente contribuir para a variabilidade no desempenho das crianças usuárias de IC". Isto é, os benéficos alcançados com o IC variam de acordo com a história de vida de cada um.

Ao discorrer sobre os benefícios do IC, pesquisadores como Capovilla (1998) Duarte e Brazorotto (2009), Tanamati, Costa e Bevilacqua (2011) pontuam que o IC, além de melhorar a percepção e produção da fala, crianças em idade escolar, com IC realizado antes dos seis anos de idade desenvolvem habilidades orais mais bem sucedidas quando comparadas com outras crianças com surdez usuárias de outros recursos tecnológicos, emitindo vogais e consoantes com entonação, ritmo e fala mais precisa, habilidades essenciais ao bom desempenho na aprendizagem escolar de leitura e escrita, por exemplo.

Devido ao aumento do número de pessoas com IC, muitas pesquisas e estudos sobre o tema têm sido realizados, e alguns pesquisadores, como Pinheiro et.al (2012) destaca que as escolas estão recebendo com mais frequência crianças implantadas, e tal fato merece ser acompanhado, estudado e divulgado, para contribuir não apenas com o sucesso do IC, mas com o sucesso escolar e a inclusão dessas crianças.

Além disso, as referidas autoras pontuam que em geral a população acredita que o IC restaura a audição, e por isso crianças com IC não apresentam dificuldades, o que não é verdade, pois como discutido anteriormente, o processo para distinção dos sons após a cirurgia, é longo e requer acompanhamento profissional para que a pessoa aprenda a ouvir, e pela audição aprenda a se comunicar. Sendo assim, dependendo do momento em que esta criança se encontra no processo de treinamento auditivo pós cirurgia, ela ainda não distingue todos os sons, logo ainda não tem uma linguagem oral estabelecida, o que torna difícil o aprendizado de outras linguagens como leitura e escrita, o que requer acompanhamento pedagógico planejado e orientado às necessidades dessa criança. É necessário investimento em desenvolvimento e divulgação de pesquisas sobre a educação de pessoas com IC, uma forma de contribuir com estudos na área, com a educação dessas pessoas e desmistificar a ideia de que o IC transforma a pessoa com surdez em ouvinte.

Considerando o cenário, ou seja, a demanda crescente de crianças com IC frequentando o ensino fundamental, e lidando com condições de ensino de diferentes tipos de linguagens (ler, escrever, ouvir, falar) é necessário investir em pesquisas desse tipo, mas sobretudo caracterizar quais são as tendências de pesquisa internacional e nacional. Sendo assim, a presente pesquisa tem como questão norteadora: Quais as tendências da pesquisa internacional e nacional sobre Implante Coclear na área da educação?

Para responder a referida questão o presente estudo teve como objetivo geral: identificar, analisar e comparar pesquisas internacionais e nacionais sobre educação de 
pessoas com IC. E como objetivo específico: conhecer os temas de pesquisas desenvolvidos em âmbito internacional e nacional.

\section{Método}

Trata-se de uma pesquisa bibliográfica, de caráter qualitativo e quantitativo, desenvolvida a partir de artigos científicos, disponíveis em bancos de dados internacional e nacional.

Pesquisas bibliográficas que utilizam publicações como fonte de informação, caso desta pesquisa, de acordo com Marconi e Lakatos (1990) podem ser desenvolvidas com base em alguns procedimentos:

I) identificação - consistiu no reconhecimento do assunto, a partir de leituras sobre o tema, fazendo um aprofundamento sobre IC a partir de autores e pesquisas de referência na área, identificando os principais bancos de dados que divulgam tais pesquisas.

II) localização - busca da fonte das publicações sobre o tema realizada nos bancos de dados selecionados - em Education Resources Information Center (ERIC) para artigos internacionais e Scientific Electronic Library Online (SCIELO) e Literatura Latino-Americana e do Caribe em Ciências da Saúde (LILACS) para artigos nacionais.

III) compilação - sistematização do material encontrado, selecionando apenas os artigos sobre IC desenvolvendo temas relacionados à educação.

IV) fichamento - transcrição dos dados nas fichas bibliográficas, destacando-se informações relevantes, como fonte e ano de publicação, autores e resumo da pesquisa realizada, os quais foram organizados por tema de pesquisa.

Tais etapas foram desenvolvidas na busca de artigos internacionais e nacionais sobre o tema educação de pessoas com implante coclear.

Com o intuito de obter um panorama atual da pesquisa sobre o tema delimitou-se a busca de artigos publicados e indexados em Bancos de Dados no período de 2009 a 2013, ou seja, nos últimos cinco anos, uma vez que há muitas pesquisas discorrendo sobre IC, devido às constantes mudanças nos recursos tecnológicos utilizados e consequentemente nas técnicas de cirurgia e resultados alcançados.

A busca dos artigos foi feita utilizando-se o termo: "Cochlear implant" e "implante coclear" para a pesquisa internacional e nacional respectivamente. 
A partir da localização dos artigos indexados realizou-se a sistematização das informações encontradas, organizando-as em fichas onde foram registrados: autor, título, ano de publicação, local de publicação e organização dos temas pesquisados.

Dentre todos os artigos encontrados foram selecionados somente aqueles que desenvolvem temas ligados à área da educação, os quais são objetos da presente pesquisa. $\mathrm{O}$ termo "educação" e ou "educação de pessoas com implante coclear" não foram um critério de busca dos artigos, uma vez que ao realizar a pesquisa com tais termos apareciam artigos sobre outros temas e algumas vezes a pesquisa resultava em nenhum artigo encontrado.

As informações coletadas foram organizadas em figuras e tabelas descrevendo os temas desenvolvidos e pontuando o número de artigos discorrendo sobre cada tema.

\section{Análise dos Resultados}

As tabelas com a descrição dos temas encontrados e denominação de tais temas foram organizados pelas pesquisadoras e submetidos à apreciação de três juízes com o intuito de aferir confiabilidade aos temas e suas respectivas descrições.

Isto é, a partir da leitura e categorização dos temas encontrados, foi preciso definir de forma que ficasse o mais claro possível aos futuros leitores em que consiste tal tema e sobre o que os artigos pertencentes ao mesmo discutem e se encaixam em determinado tema e não em outros.

Assim, após a nomeação e descrição das categorias encontradas, as tabelas foram enviadas a três pessoas com conhecimento e experiência na área de pesquisas e trabalhos sobre implante coclear, para que elas analisassem, corrigissem e complementassem tais temas, de modo a deixa-los o mais objetivo e coerente possível.

Somente, após a apreciação dos três juízes as pesquisadoras deram continuidade a escrita da pesquisa.

\section{Resultados}

A busca de artigo em ERIC, SCIELO e LILACS, resultou em: 


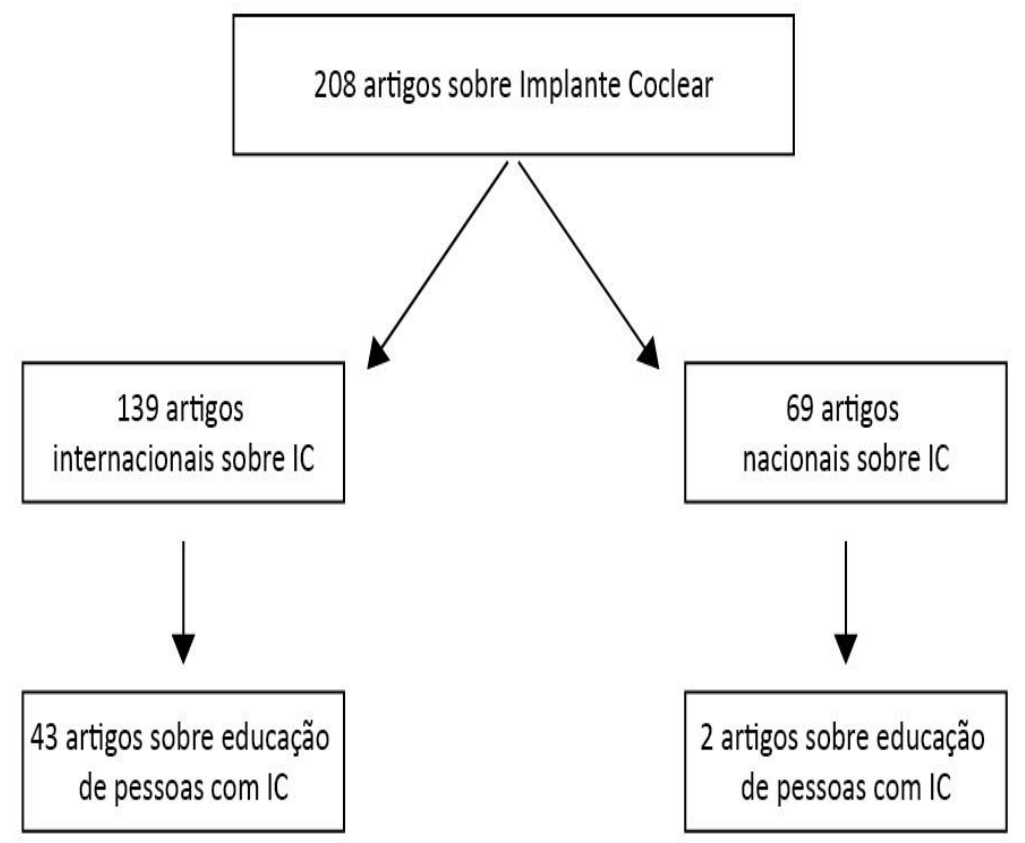

A busca de artigos internacionais em ERIC resultou em um total de 139 artigos publicados e indexados nos últimos anos, conforme representado na figura 1.

Figura 1 - Número de artigos internacionais sobre IC de 2009 a 2013.

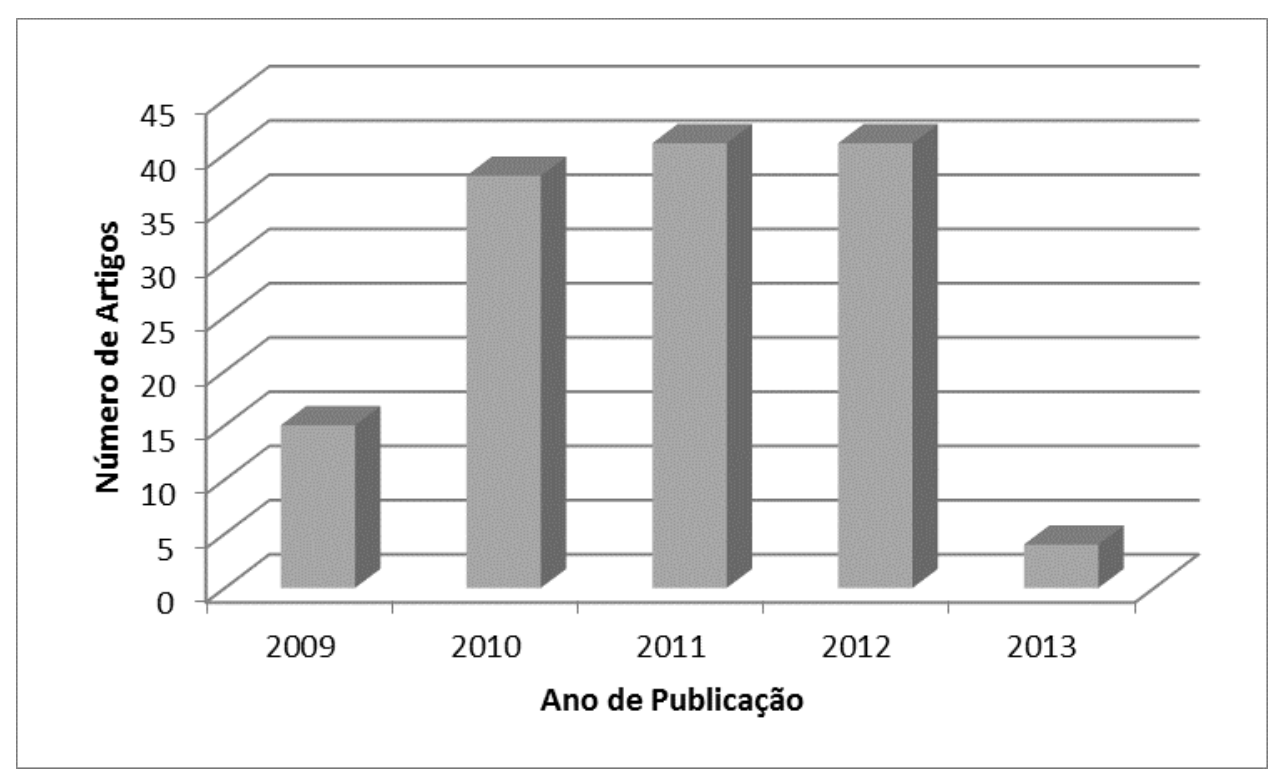

A partir da figura 1, é possível observar que o número de publicação sobre IC tem aumentado ao longo dos anos, tendo número de publicações igual em 2011 e 2012. 
Os artigos indexados no período foram publicados em diferentes periódicos, tanto da área da saúde como da educação, como é possível visualizar a partir da Tabela 1.

Tabela 1 - Número de artigos publicados em periódicos internacionais

\begin{tabular}{|c|c|}
\hline Periódico de indexação & $\begin{array}{l}\text { Número de } \\
\text { artigos }\end{array}$ \\
\hline Journal of deaf studies and deaf education & 28 \\
\hline Journal of speech, language and hearing research & 28 \\
\hline Deafness and education international & 15 \\
\hline Clinical linguistics \& Phonetics & 10 \\
\hline Volta Review & 7 \\
\hline American annals of the deaf & 5 \\
\hline Communication Disorders quarterly & 3 \\
\hline International Journal of Bilingual education and Bilinguism & 3 \\
\hline Journal of Communication Disorders & 3 \\
\hline Odyssey: New Directions in deaf education & 3 \\
\hline Research in Developmental Disabilities: a multidisciplinary journal & 3 \\
\hline Applied Psycholinguistics & 2 \\
\hline Developmental Science & 2 \\
\hline International Journal of language \& Communication & 2 \\
\hline American Journal of Speech language pathology & 1 \\
\hline Brain: a journal of neurology & 1 \\
\hline Developmental Medicine \& Child Neurology & 1 \\
\hline Disability \& Society & 1 \\
\hline Discourse: Studies in the cultural politics of education & 1 \\
\hline Early Child Development and care & 1 \\
\hline European Journal of Psychology of education & 1 \\
\hline
\end{tabular}




\begin{tabular}{|c|c|}
\hline European Journal of Special needs education & 1 \\
\hline IEEE Transaction on Education & 1 \\
\hline Journal of Access Services & 1 \\
\hline Journal of experimental Psychology applied & 1 \\
\hline $\begin{array}{l}\text { Journal of experimental Psychology: human perception and } \\
\text { performance }\end{array}$ & 1 \\
\hline Journal of multilingual and multicultural Development & 1 \\
\hline Journal of the international association of special education & 1 \\
\hline Language and Speech & 1 \\
\hline Language Policy & 1 \\
\hline Language Sciences & 1 \\
\hline Language, Speech and hearing services in school & 1 \\
\hline Mind, Brain and education & 1 \\
\hline Music Education Research & 1 \\
\hline Music Educators Journal & 1 \\
\hline Qualitative Inquiry & 1 \\
\hline Scientific studies of reading & 1 \\
\hline Support of learning & 1 \\
\hline Turkish online Journal of qualitative inquiry & 1 \\
\hline
\end{tabular}

A partir dos títulos de indexação dos periódicos encontrados no período é possível perceber que o tema implante coclear tem sido foco de pesquisa não apenas na área da saúde, o que se comprova a partir dos periódicos da área de fonoaudiologia e medicina, mas também na área de psicologia, linguística, educação e arte, como é o caso dos periódicos de música. Há número expressivo de periódicos que contém em seu título o termo Educação, o que demonstra que há muitas publicações internacionais sobre IC sendo divulgadas para leitores dessa área.

Dentre o total de artigos internacionais sobre IC destacou-se os artigos que discorrem sobre a educação de pessoas com IC, como representado na figura 2. 
Figura 2 - Porcentagem de artigos internacionais sobre educação de pessoas com IC.

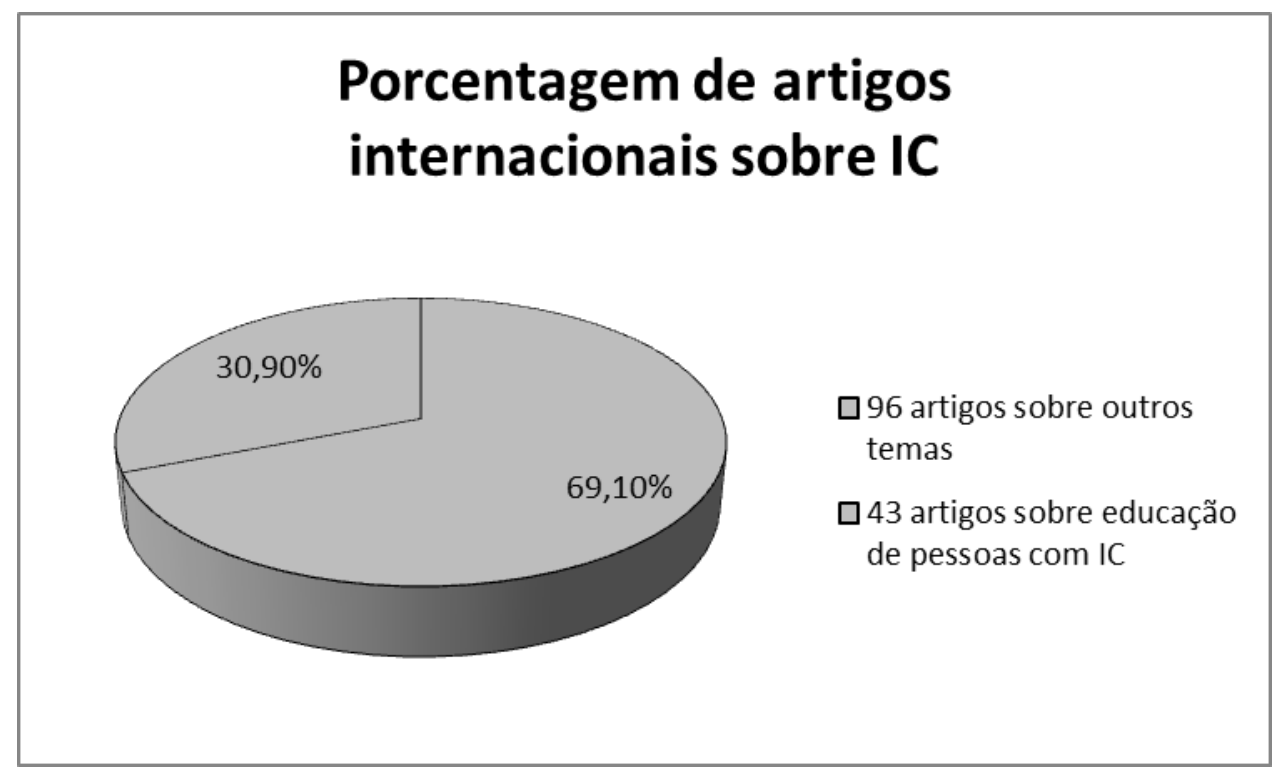

Os artigos internacionais que abordam o tema educação de pessoas com IC foram lidos e organizados de acordo com tema estudado. Os temas encontrados no período analisado e o número de pesquisas desenvolvendo cada tema estão descritos na tabela 2.

Tabela 2 - Descrição dos temas dos artigos internacionais sobre Educação de pessoas com IC

\begin{tabular}{|c|c|c|}
\hline Tema & Descrição & Número de artigos \\
\hline Leitura & 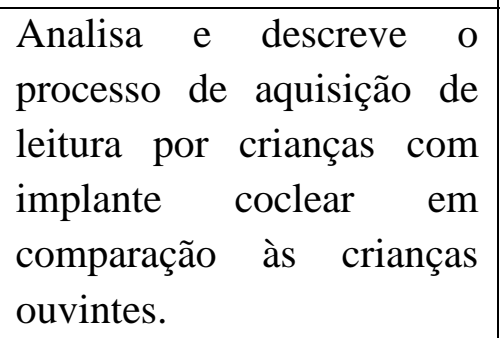 & 9 \\
\hline $\begin{array}{l}\text { Educação de Surdos com } \\
\text { Implante Coclear }\end{array}$ & 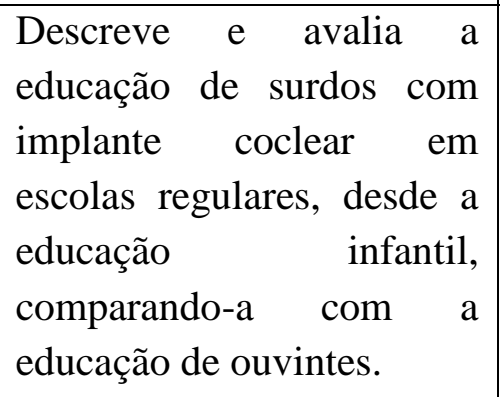 & 8 \\
\hline $\begin{array}{l}\text { Educação musical de } \\
\text { Crianças com Implante }\end{array}$ & \begin{tabular}{lcc} 
Descreve & \multicolumn{2}{c}{ atividades } \\
musicais & realizadas & com \\
crianças & surdas & com
\end{tabular} & 5 \\
\hline
\end{tabular}




\begin{tabular}{|c|c|c|}
\hline Coclear & $\begin{array}{lr}\text { implante coclear e } & \text { a } \\
\text { importância destas ao } & \text { a } \\
\text { desenvolvimento } & \text { e } \\
\text { aprendizagem da criança. }\end{array}$ & \\
\hline Estímulo visual & $\begin{array}{l}\text { Investiga a importância de } \\
\text { estímulos visuais ao ensino } \\
\text { e a aprendizagem de } \\
\text { crianças surdas com } \\
\text { implante coclear. }\end{array}$ & 4 \\
\hline $\begin{array}{l}\text { Habilidade para contar } \\
\text { histórias }\end{array}$ & $\begin{array}{l}\text { Analisa a capacidade de } \\
\text { crianças com implante } \\
\text { coclear em contar histórias, } \\
\text { respeitando a cronologia, a } \\
\text { sequência dos fatos. }\end{array}$ & 3 \\
\hline Inclusão & $\begin{array}{l}\text { Descreve como se dá a } \\
\text { inclusão de crianças surdas } \\
\text { com implante coclear no } \\
\text { ensino regular. }\end{array}$ & 3 \\
\hline $\begin{array}{l}\text { Formação de professores } \\
\text { de crianças com IC }\end{array}$ & $\begin{array}{l}\text { Analisa a formação inicial } \\
\text { e o preparo de professores } \\
\text { para ensinar crianças com } \\
\text { implante coclear. }\end{array}$ & 3 \\
\hline Alfabetização & $\begin{array}{l}\text { Descreve e analisa o } \\
\text { processo de alfabetização } \\
\text { da criança com implante } \\
\text { coclear em comparação à } \\
\text { criança ouvinte. }\end{array}$ & 1 \\
\hline $\begin{array}{l}\text { Comparação numérica } \\
\text { verbal e não verbal }\end{array}$ & $\begin{array}{l}\text { Avalia o } \quad \text { uso de } \\
\text { comparação e } \\
\text { na relação }\end{array}$ & 1 \\
\hline Escolas Especiais & $\begin{array}{l}\text { Descreve os serviços } \text { e } \\
\text { educação oferecidos por } \\
\text { uma escola especial de } \\
\text { surdos para crianças com } \\
\text { implante coclear. }\end{array}$ & 1 \\
\hline
\end{tabular}




\begin{tabular}{|c|c|c|}
\hline Escrita & $\begin{array}{l}\text { Analisa o desenvolvimento } \\
\text { da escrita e a ortografia de } \\
\text { crianças surdas com I.C }\end{array}$ & 1 \\
\hline $\begin{array}{l}\text { Graduação para } \\
\text { intervenção oral precoce }\end{array}$ & $\begin{array}{l}\text { Descreve o currículo e as } \\
\text { perspectivas da } \\
\text { implantação de um curso } \\
\text { de Graduação para } \\
\text { intervenção oral precoce da } \\
\text { Universidade do Mississipi } \\
\text { e sua importância no } \\
\text { atendimento das } \\
\text { necessidades de pessoas } \\
\text { com implante coclear. }\end{array}$ & 1 \\
\hline $\begin{array}{l}\text { Preparação acadêmica de } \\
\text { pessoas com implante } \\
\text { coclear para a faculdade }\end{array}$ & $\begin{array}{l}\text { Descreve um serviço de } \\
\text { acompanhamento } \\
\text { orientação educacional à } \\
\text { jovens com implante } \\
\text { coclear com o intuito de } \\
\text { prepara-los para a } \\
\text { faculdade. }\end{array}$ & 1 \\
\hline Serviços de biblioteca & $\begin{array}{l}\text { Descreve } \\
\text { desenvolvimento de um } \\
\text { serviço de biblioteca } \\
\text { oferecido exclusivamente à } \\
\text { pessoas com implante } \\
\text { coclear. }\end{array}$ & 1 \\
\hline $\begin{array}{l}\text { TDAH }^{1} \text { em crianças com } \\
\text { implante coclear }\end{array}$ & $\begin{array}{lr}\text { Relata casos de } & \text { crianças } \\
\text { com implante } & \text { coclear } \\
\text { diagnosticadas } & \text { com } \\
\text { TDAH. } & \end{array}$ & 1 \\
\hline \multicolumn{3}{|l|}{ Total } \\
\hline
\end{tabular}

Dos temas relacionados a educação, destacam-se "Leitura" e "Educação de surdos com Implante Coclear", com nove e oito artigos respectivamente, demonstrando que em

\footnotetext{
${ }^{1}$ Transtorno de Déficit de Atenção e Hiperatividade.
} 
muitos países há uma preocupação e cuidado com a aquisição da leitura de pessoas com IC, assim como sua educação em geral, desde a educação infantil até o ensino superior, com foco para as habilidades essenciais a educação dessas pessoas, como alfabetização, leitura, escrita, conhecimento matemático, educação especial e com os profissionais que lidam com tais pessoas, os professores, além dos serviços e atendimentos oferecidos à essa população, outros temas identificados, ou seja, há pesquisas sobre todos os aspectos, peculiaridades e serviços importantes à educação da pessoa com IC.

Os artigos internacionais desenvolvendo os temas relacionados à área de educação estão sistematizados na Tabela 3.

Tabela 3 - Artigos internacionais desenvolvendo temas relacionados à educação.

\begin{tabular}{|c|c|}
\hline Tema & $\begin{array}{l}\text { Lista de Referências dos artigos internacionais relacionados } \\
\text { à educação. }\end{array}$ \\
\hline & $\begin{array}{l}\text { DILLON, C.M.; JONG,K.;PISONI,D.B. Phonological } \\
\text { Awareness, Reading Skills, and Vocabulary Knowledge in } \\
\text { Children Who Use Cochlear Implants. Journal of Deaf Studies } \\
\text { and Deaf Education. Apr 2012; 17(2): 205-226, } 2012 .\end{array}$ \\
\hline & $\begin{array}{l}\text { WOLL, BENCIE. Speech Reading Revisited. Deafness and } \\
\text { Education International. v14 n1 p16-21 Mar, } 2012 .\end{array}$ \\
\hline & $\begin{array}{l}\text { BOUTON, S.; BERTONCINI, J.; SERNICLAES, W.; COLÉ, } \\
\text { P. Reading and Reading-Related Skills in Children Using } \\
\text { Cochlear Implants: Prospects for the Influence of Cued Speech. } \\
\text { Journal of Deaf Studies and Deaf Education. v16 n } 4 \text { p458-473, } \\
2011 \text {. }\end{array}$ \\
\hline & $\begin{array}{l}\text { HAYES, H.; TREIMAN, R. Spelling in Deaf Children with } \\
\text { Cochlear Implants: Implications for Instruction. Scientific } \\
\text { studies of reading. v15 n6 p522-540, } 2011 \text {. }\end{array}$ \\
\hline & $\begin{array}{l}\text { HOOGMOED, ANNE H. VAN; VERHOEVEN, LUDO; } \\
\text { SCHREUDER, ROBERT; KNOORS, HARRY. Morphological } \\
\text { sensitivity in deaf readers of Dutch. Applied Psycholinguistics, } \\
\text { v32 n3 p619-634, 2011. }\end{array}$ \\
\hline \multirow[b]{2}{*}{ Leitura } & $\begin{array}{l}\text { HARRIS, MARGARET; TERLEKTSI, EMMANOUELA } \\
\text { Reading and Spelling Abilities of Deaf Adolescents with } \\
\text { Cochlear Implants and Hearing Aids. Journal of Deaf Studies } \\
\text { and Deaf Education. v16 n1 p24-34, 2011. }\end{array}$ \\
\hline & $\begin{array}{l}\text { JOHNSON, C.; GOSWAMI, U. Phonological awareness, } \\
\text { vocabulary, and reading in deaf children with cochlear implants. } \\
\text { Journal of Speech, language and hearing research. v53 n2 p237- } \\
261 \text { Apr } 2010 .\end{array}$ \\
\hline
\end{tabular}


JAMES, D.; RAJPUT, K.; BRINTON, J.;GOSWAMI, U. Orthographic Influences, Vocabulary Development, and Phonological Awareness in Deaf Children Who Use Cochlear Implants. Applied Psycholinguistics, v30 n4 p659-684 Oct2009.

SPENCER, L.J.; TOMBLIN, J.B. Evaluating phonological processing skills in children with prelingual deafness who use cochlear implants. Journal of Deaf Studies and Deaf Education, v14 n1 p1-21 Win2009.

ARCHBOLD, S. MAYER, C. CHUTE, P.M. Deaf Education: The Impact of Cochlear Implantation? College Experience for Young Adults with Hearing Loss, v1 n1 s/p. 2012.

CHUTE, P.M. College Experience for Young Adults with Hearing Loss. Deafness and Education International, v14 n1 p60-65 Mar 2012.

Educação de Surdos

DENHAM, P.J.; BATTRO, A.M. Education of the Deaf and Hard of Hearing in the Digital Era. Mind, Brain, and Education, v6 n1 p51-53 Mar 2012.

com IC

DE RAEVE, L.; LICHTERT, G. Changing Trends within the Population of Children Who Are Deaf or Hard of Hearing in Flanders (Belgium): Effects of 12 Years of Universal Newborn Hearing Screening, Early Intervention, and Early Cochlear Implantation. Volta Review, v112 n2 p131-148 Sum2012.

MARSCHARCK, M.; BULL, R.; SAPERE, P.; NORDMANN, E.; SKENE, W.; LUKOMSKI, J.; LUMSDEN,S. Do You See What I See? School Perspectives of Deaf Children, Hearing Children, and Their Parents European Journal of Special Needs Education, v27 n4 p483-4972012.

NUSSBAUM, D.B.; SCOTT, S. The why and how of an ASH English bimodal bilingual program. Odyssey: new directions in deaf education, v13 p14-19 2012.

Educação de Surdos com IC

VERMEULEN, A.; DE RAEVE, L.; LANGEREIS, M.; SNIK, Ad. Changing Realities in the Classroom for Hearing-Impaired Children with Cochlear Implant. Deafness and Education International, v14 n1 p36-47 Mar2012.

HAYES, H. Washington University School of Medicine: A Distinctive Program in Deaf Education Studies at the Program in Audiology and Communication Sciences (PACS). Volta Review, v110 n2 p271-278 Sum2010. 


\begin{tabular}{l|l}
\hline & and hearing research, v55 n3 p800-810 Jun2012. \\
\cline { 2 - 3 } Educação musical & $\begin{array}{l}\text { KONG, Y.Y.; MULLANGI, A.; MAROZEAU, J.; EPSTEIN, } \\
\text { M. Temporal and spectral cues for musical timbre perception in } \\
\text { electric hearing. Journal of Speech, language and hearing } \\
\text { research. v54 n3 p981-994 Jun 2011. }\end{array}$ \\
\cline { 2 - 2 } Implante Coclear & $\begin{array}{l}\text { YENNARI, M. Beginnings of song in young deaf children } \\
\text { using cochlear implants: the song they move, the song they feel, } \\
\text { the song they share. Music Education Research, v12 n3 p281- } \\
\text { 297 Sep 2010. }\end{array}$ \\
\hline
\end{tabular}

SCHRAER-JOINER, L.;CHEN-HAFTECK, L. The responses of preschoolers with $\mathrm{CI}$ to musical activities: a multiple case study. Early child Development and Care, v179 n6 p785-798 Aug 2009.

SCHRAER-JOINER, L.; PRAUSE-WEBER, M. Strategies for Working with Children with Cochlear Implants. Music Educators Journal, v96 n1 p48-55, 2009.

VALKENIER, B.; DUYNE, J.Y.; ANDRINGA, T.C.; A BAŞKENT, D. Audiovisual Perception of Congruent and Incongruent Dutch Front Vowels. Journal of Speech, Language, and Hearing Research, v55 n6 p1788-1801 Dec 2012.

\begin{tabular}{l|l} 
Estímulo Visual & WAYNE, R.V.; JOHNSRUDE, I.S. The role of visual speech
\end{tabular} information in supporting perceptual learning of degraded speech. Journal of Experimental Psycology applied. v18 n4 p419-435 Dec 2012.

PILLING, M.; THOMAS, S. Audiovisual cues and perceptual learning of spectrally distorted speech. Language and Speech, vol. 54 no. 4 487-497, Dec 2011.

\begin{tabular}{l|l}
\cline { 2 - 3 } Estímulo Visual & NEUMEYER, V.; HARRINGTON, J.; DRAXLER, C. An
\end{tabular} acoustic analysis of the vowel space in young and old cochlearimplant speakers. Clinical Linguistics \& Phonetics, v24 n9 p734-741 Sep 2010.

HUTTUNEN, K.; RYDER, N. How children with normal hearing and children with a cochlear implant use mentalizing vocabulary and other evaluative expressions in their narratives. Clinical Linguistics \& Phonetics, v26 n10 p823-844 Oct 2012.

Habilidade para \begin{tabular}{l} 
INGBER, S.; EDEN, S. Enhancing sequential time perception \\
\cline { 2 - 2 } Contal
\end{tabular} Contar Histórias $\quad$ and storytelling ability of deaf and hard of hearing children. American Annals of the deaf, v156 n4 p391-401 2011.

WORSFOLD, S.; MAHON, M.; YUEN, H.M.; KENNEDY, C. Narrative skills following early confirmation of permanent 


\begin{tabular}{|c|c|}
\hline & $\begin{array}{l}\text { childhood hearing impairment. Developmental Medicine \& } \\
\text { Child Neurology. v52 n10 p922-928 Oct } 2010 .\end{array}$ \\
\hline \multirow{3}{*}{ Inclusão } & $\begin{array}{l}\text { REKKEDAL, A.M. Assistive Hearing Technologies Among } \\
\text { Students With Hearing Impairment: Factors That Promote } \\
\text { Satisfaction. Journal of Deaf Studies and Deaf Education, v17 } \\
\text { n4 p499-517 Fall 2012. }\end{array}$ \\
\hline & $\begin{array}{l}\text { PUNCH, R.; HYDE, M. Social participation of children and } \\
\text { adolescents with cochlear implants: a qualitative analysis of } \\
\text { parent, teacher, and child interviews. Journal of Deaf Studies } \\
\text { and Deaf Education, v16 n4 p474-493 Fall } 2011 \text {. }\end{array}$ \\
\hline & $\begin{array}{l}\text { JACHOVA, Z; KOVACEVIC, J. Cochlear implants in the } \\
\text { inclusive classroom: a case study. Supporting for learning, v25 } \\
\text { n1 p33-37 Feb 2010. }\end{array}$ \\
\hline \multirow{3}{*}{$\begin{array}{l}\text { Formação } \\
\text { professores } \\
\text { crianças com IC }\end{array}$} & $\begin{array}{l}\text { PUNCH, R.; HYDE, M. Children With Cochlear Implants in } \\
\text { Australia: Educational Settings, Supports, and Outcomes. } \\
\text { Journal of Deaf Studies and Deaf Education. v15 n4 p405-421 } \\
\text { Fall 2010. }\end{array}$ \\
\hline & $\begin{array}{l}\text { LINDEMAN, K.W. For information and fellowship: Deaf club } \\
\text { eases isolation. Odyssey: Directions in Deaf Education. v10 n1 } \\
\text { p20-22 Spr-Sum } 2009 \text {. }\end{array}$ \\
\hline & $\begin{array}{l}\text { WILSON, K.; NEVINS, M.E.; HOUSTON, K.T. Professional } \\
\text { Development for In-Service Practitioners Serving Children who } \\
\text { are Deaf \& Hard of Hearing. Volta Review, v110 n2 p231-247 } \\
\text { Sum } 2010 .\end{array}$ \\
\hline Alfabetização & $\begin{array}{l}\text { DESJARDIN, J.L.; AMBROSE, S.E.; EISENBERG, L.S. } \\
\text { Maternal Involvement in the Home Literacy Environment: } \\
\text { Supporting Literacy Skills in Children With Cochlear Implants. } \\
\text { Communication Disorders Quarterly, v32 n3 p135-150 May } \\
\text { 2011. }\end{array}$ \\
\hline $\begin{array}{l}\text { Comparação } \\
\text { numérica verbal e } \\
\text { não verbal }\end{array}$ & $\begin{array}{l}\text { ARFÉ, B.; LUCANGELI, D.G.; MONZANI, D.; } \\
\text { GUBERNALE, M.; TREVISI, P. Analogic and symbolic } \\
\text { comparison of numerosity in preschool children with cochlear } \\
\text { implants. Deafness and Education International, v13 n1 p34-45 } \\
\text { Mar 2011. }\end{array}$ \\
\hline Escolas Especiais & $\begin{array}{l}\text { DE RAEVE, L.; BAERTS, J.; COLLEYE, E. ; CROUX, E. } \\
\text { Changing Schools for the Deaf: Updating the Educational } \\
\text { Setting for Our Deaf Children in the } 21 \text { st Century, a Big } \\
\text { Challenge. Deafness and Education International, v14 n1 p } 48 \\
59 \text { Mar } 2012 \text {. }\end{array}$ \\
\hline
\end{tabular}




\begin{tabular}{|c|c|}
\hline Escrita & $\begin{array}{l}\text { ASKER-ANÁRSON, L.; IBERTSSON, T.; WASS, M.; } \\
\text { WENGELIN, A.; SAHLÉN, B. Picture-Elicited Written } \\
\text { Narratives, Process and Product, in } 18 \text { Children With Cochlear } \\
\text { Implants. Communication Disorders Quarterly, v31 n4 p195- } \\
212 \text { 2010. }\end{array}$ \\
\hline $\begin{array}{ll}\text { Graduação } & \text { para } \\
\text { intervenção } & \text { oral } \\
\text { precoce } & \end{array}$ & $\begin{array}{l}\text { PERIGOE, C.B.; TELLER, H. The University of Southern } \\
\text { Mississippi: Developing a State-of-the-Art Graduate Program in } \\
\text { Early Oral Intervention. Volta Review, v110 n2 p315-322 Sum } \\
2010 \text {. }\end{array}$ \\
\hline $\begin{array}{l}\text { Preparação } \\
\text { acadêmica } \\
\text { pessoas de } \\
\text { implante com } \\
\text { para a faculdade }\end{array}$ & $\begin{array}{l}\text { CONVERTINO, C.M.; MARSCHACK, M.; SAPERE, P.; } \\
\text { SARCHET, T.; ZUPAN, M. Predicting Academic Success } \\
\text { Among Deaf College Students, Journal of Deaf Studies and } \\
\text { Deaf Education, v14 n3 p324-343 Sum } 2009 .\end{array}$ \\
\hline $\begin{array}{l}\text { Serviços } \\
\text { Biblioteca }\end{array}$ & $\begin{array}{l}\text { RILEY, C. Training for Library Patrons Who Are Hard of } \\
\text { Hearing. Journal of Access Services, v6 n1-2 p72-972009. }\end{array}$ \\
\hline $\begin{array}{l}\text { TDAH em crianças } \\
\text { com implante } \\
\text { coclear }\end{array}$ & $\begin{array}{l}\text { MORENO-TORRES, I.; TORRES, S.; SANTANA, R. Lexical } \\
\text { and grammatical development in a child with cochlear implant } \\
\text { and attention deficit: A case study. Clinical Linguistics \& } \\
\text { Phonetics, v24 n9 p706-721 Sep 2010. }\end{array}$ \\
\hline
\end{tabular}

Com base nos temas de pesquisas internacionais encontrados é possível perceber que a efetividade e sucesso do implante coclear requerem também atenção e investimento na educação dessas pessoas, complementado o atendimento e serviços de manutenção do IC e serviço fonoaudiológico para desenvolvimento da fala.

O panorama da pesquisa internacional sobre o tema leva a uma reflexão sobre a pesquisa nacional sobre implante coclear. Para conhecer as pesquisas nacionais sobre implante coclear realizou-se uma revisão da literatura nacional.

A busca de artigos publicados e indexados no período analisado em LILACS e SCIELO resultou em 69 artigos, conforme representado na figura 3. 
Figura 3 - Número de artigos nacionais sobre IC de 2009 a 2013.

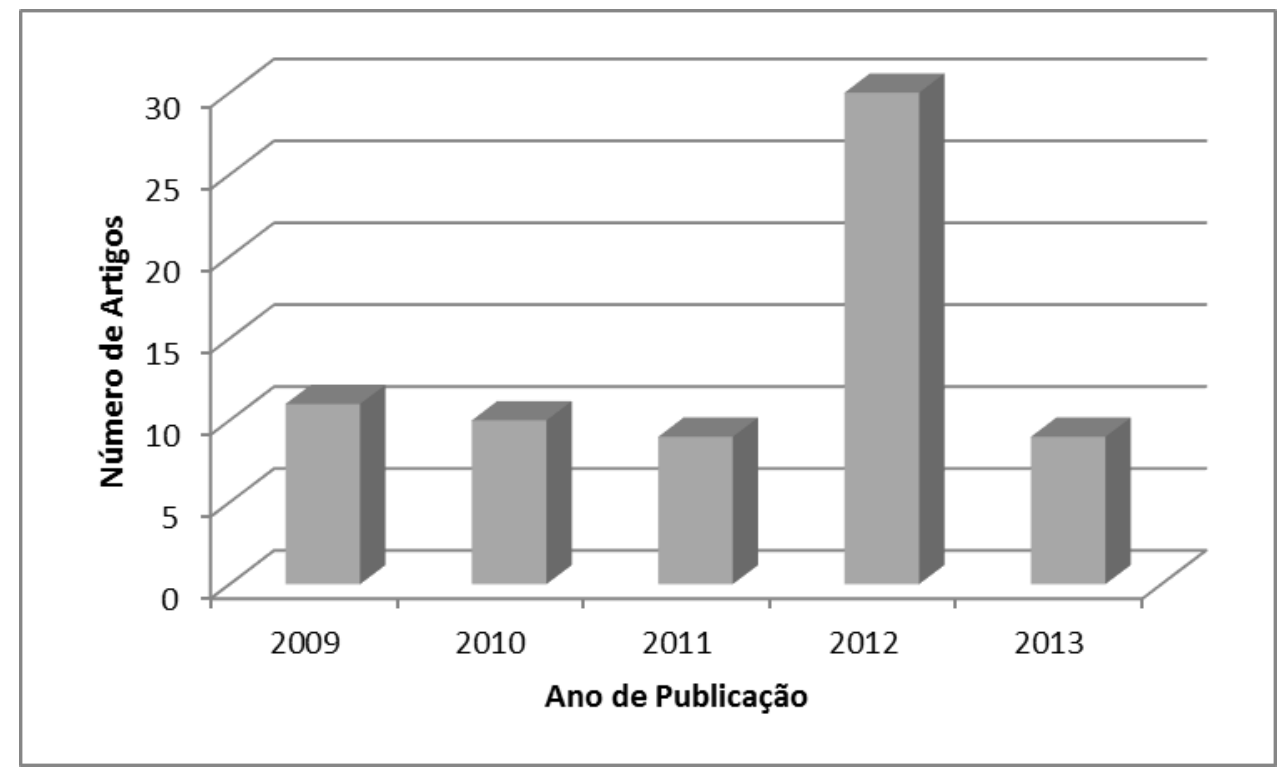

Dentre o total de artigos nacionais sobre IC destacou-se os artigos que discorrem sobre a educação de pessoas com IC, como representado na figura 4.

Figura 4 - Porcentagem de artigos nacionais sobre educação de pessoas com IC.

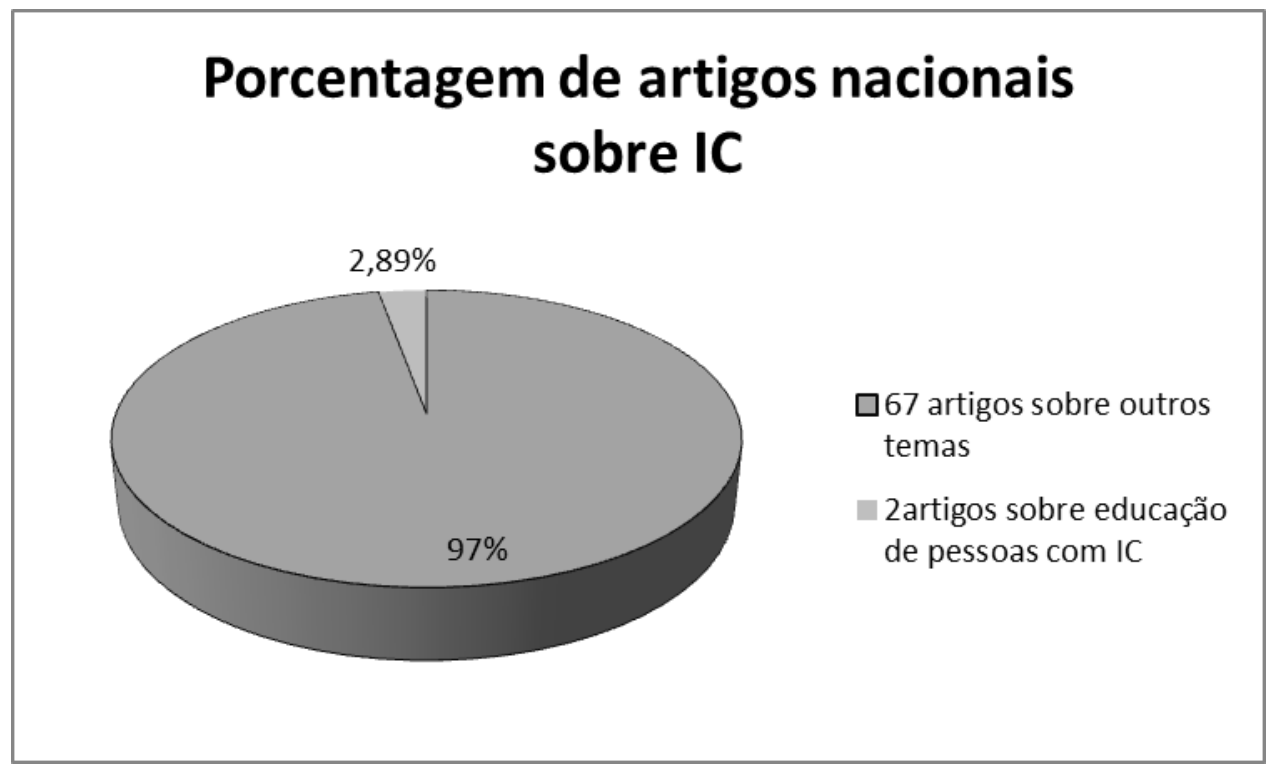

Os artigos nacionais que abordam o tema educação de pessoas com IC foram lidos e organizados de acordo com o tema estudado. Os temas encontrados no período analisado e o número de pesquisas desenvolvendo cada tema estão descritos na tabela 4 . 
Tabela 4 - Descrição dos temas dos artigos nacionais sobre Educação de pessoas com IC

\begin{tabular}{l|l|l}
\hline Tema & Descrição & Número de Artigos \\
\hline Desempenho Escolar & $\begin{array}{l}\text { Avalia e compara o } \\
\text { desempenho escolar de } \\
\text { crianças com I.C às } \\
\text { crianças ouvintes. }\end{array}$ & 1 \\
\hline Escrita & $\begin{array}{l}\text { Analisa o desenvolvimento } \\
\text { da escrita e a ortografia de } \\
\text { crianças surdas com I.C }\end{array}$ & 1 \\
\hline & \multicolumn{3}{l}{} \\
\hline
\end{tabular}

Os artigos nacionais desenvolvendo temas ligados à educação estão sistematizados na tabela 5 .

Tabela 5 - Artigos nacionais desenvolvendo temas relacionados à Educação

\begin{tabular}{c|l}
\hline Tema & $\begin{array}{l}\text { Lista de Referência de artigos nacionais relacionados } \\
\text { à educação }\end{array}$ \\
\hline Desempenho escolar & $\begin{array}{l}\text { PINHEIRO, A. B. S. M.; YAMADA, M. O.; } \\
\text { BEVILACQUA, M. C.; CRENITTE, P. A. P. Avaliação } \\
\text { das habilidades escolares de crianças com implante } \\
\text { coclear. Revista CEFAC, São Paulo, vol.14 no.5, 2012. }\end{array}$ \\
\hline Escrita & $\begin{array}{l}\text { DUARTE, J. L.; BRAZOROTTO, J. S. Análise das } \\
\text { estratégias utilizadas em um grupo terapêutico } \\
\text { pedagógico para auxiliar o desenvolvimento da } \\
\text { linguagem escrita em crianças com deficiência auditiva. } \\
\text { Revista Brasileira de Educação Especial, Marília, v. } \\
15, \text { n. 3,2009. }\end{array}$ \\
\hline
\end{tabular}

Este levantamento revela uma tendência de pesquisa na área, demonstrando a escassez de pesquisas nacionais discorrendo sobre educação, inclusão e atendimento pedagógico de pessoas com IC, o que deveria ser foco de pesquisa, uma vez que tal atendimento está previsto como um dos requisitos para realização do IC, conforme discutido anteriormente. 
Isto é, os Centros/ núcleos que realizam a cirurgia precisam ter uma equipe de profissionais que acompanhem e conduzam a adaptação da pessoa ao implante coclear, pois caso contrário a pessoa não se beneficiará com a cirurgia, e dentre os serviços previstos para o sucesso do IC está o atendimento educacional. E o trabalho realizado com essas pessoas precisa ser divulgado em pesquisas descrevendo o acompanhamento e a aprendizagem de habilidades auditivas e de linguagem oral e escrita, assim como já acontece em outros países.

O levantamento realizado pontua que há temas de pesquisa sobre IC que precisam ser aprofundados, como acontece em estudos internacionais, como exemplo, a inclusão desses alunos no ensino regular, o tipo de atendimento educacional especializado que necessitam, como tal atendimento é oferecido, a relação entre o professor e estes alunos em sala de aula: as dificuldades dos professores em ensinar estes alunos, e as dificuldades encontras por estes alunos para aprender os conteúdos curriculares básicos, como leitura, escrita e operações matemáticas, foco da presente pesquisa. Temas que precisam ser explorados, e ficam como sugestão para pesquisas futuras, além de justificar a importância do presente trabalho ao contribuir com a pesquisa nacional na área de Implante Coclear.

\section{Discussão}

A busca por artigos internacionais sobre IC demonstra que este tema tem sido bastante pesquisado nos últimos anos, pois há um número relevante de artigos publicados todos os anos.

Dentre os 139 artigos internacionais sobre IC, 30.9\% tratam de temas relacionados à educação, ou seja, 43 artigos. Número expressivo, que demonstra a preocupação com a temática e o consequente investimento em pesquisas na área, uma vez que a maioria deles descreve experiência de ensino e aprendizagem e processo educacional desenvolvido com pessoas surdas com IC, uma forma de divulgar o atendimento pós implante e os benefícios trazidos com as intervenções realizadas.

Há um número significativo de pesquisas internacionais discorrendo sobre a leitura e a educação de crianças com IC, temas com maior número de pesquisas publicadas, divulgando exemplos de atendimento educacional realizado em escolas regulares para desenvolvimento da leitura, comparando o processo de aquisição entre crianças surdas e ouvintes, as dificuldades e recursos utilizados, pois como defendem Duarte e Brazorotto (2009) a criança surda com IC em processo de educação escolar precisa de grande variedade de recursos para impulsionar a construção de sua leitura e escrita, enquanto ainda está construindo e aprimorando sua linguagem oral.

A educação musical e o uso de estímulos visuais como recursos no aprendizado de crianças com IC são temas com número de pesquisas acentuado, demonstrando que na educação das crianças com IC há diversas possiblidades de trabalho e intervenção que podem 
contribuir com o processo de educação. Dentre as pesquisas do tema "Educação Musical de crianças com Implante Coclear" há desde relatos de práticas de ensino de música, destacando a distinção e percepção de diferentes sons por pessoas com IC, até práticas de trabalho com crianças de educação infantil por meio de atividades musicais, uma vez que a música está muito presente no cotidiano das pessoas, em especial nas práticas escolares da educação infantil, e o uso do IC, permite às crianças dessa faixa etária participar, interagir e aprender a partir de tais propostas. Como os sons produzidos pelo IC diferem da audição normal, e certos sons e até músicas, a princípio soam como ruídos, tornando-se natural após trabalho de reabilitação auditiva pós cirurgia, é essencial parceria entre equipe de profissionais da saúde e equipe pedagógica, complementando o trabalho realizado em benefício do sucesso da pessoa com IC (TANAMATI; COSTA; BEVILACQUA, 2011).

Já o tema "Estímulo Visual" traz pesquisas evidenciando o quanto o uso de recursos visuais, como figuras e objetos podem contribuir com o aprendizado da pessoa com IC, não apenas na aquisição da linguagem oral, mas de diversos conteúdos curriculares, perspectiva também evidenciada por Duarte e Brazorotto (2009).

Além dos referidos temas há artigos internacionais discorrendo sobre a inclusão e escolas especiais para pessoa com IC, evidenciando que o debate atual sobre o melhor paradigma educacional - com foco no desenvolvimento da linguagem oral (oralismo) ou na língua de sinais (bilinguismo), ou na educação regular junto com outras crianças surdas e ouvintes (inclusão) ou em escolas especiais com crianças surdas - também se faz presente na realidade de pessoas surdas com IC. Como discute Capovilla (1998) tano o IC, quanto a língua de sinais são alternativas complementares na educação da criança surda, e precisam estar presentes também no debate sobre o melhor paradigma educacional para a criança surda com IC, envolvendo os profissionais que atendem essas crianças, a família e a equipe escolar.

Ainda dentre os artigos internacionais encontrados há pesquisas sobre a formação de professores que atuam com crianças surdas com IC, o que demonstra a importância destes profissionais na educação dessas crianças, uma vez que é imprescindível que eles obtenham informações sobre IC e conheçam formas de atuação, de ensino e práticas docentes que respeitem e atendam às necessidades da criança implantada ainda em sua formação, preparando-os para a prática.

Os temas: alfabetização, escrita e habilidade para contar histórias demonstram que os anos iniciais de escolarização de crianças com IC são foco de pesquisa, e as crianças implantadas precisam de acompanhamento e atenção às suas necessidades e dificuldades como qualquer outra criança, como recomendado pela portaria que regulamenta o IC no Brasil (BRASIL, 1999) e por pesquisadores da área, como Pinheiro et al (2012).

Alguns dos temas encontrados são específicos, mas comprovam o investimento na área da educação de pessoas com IC, como é caso dos temas: Graduação para intervenção precoce, descrevendo a implantação de um curso voltado para formação de profissionais para 
trabalhar com pessoas com IC, atendendo suas dificuldades e necessidades; Serviços de biblioteca, com a descrição de um atendimento especializado a pessoas com IC.

As pesquisas internacionais estão tão avançadas que já há artigo investigando a associação da surdez a transtornos globais, no caso o TDAH.

No Brasil, a pesquisa sobre IC parece estar um pouco aquém das pesquisas internacionais, pois dentre os 69 artigos encontrados sobre IC, somente dois artigos, aproximadamente $2,89 \%$ das pesquisas encontradas no período, desenvolvem temas relacionados a educação, sendo um artigo sobre o Desempenho escolar de crianças com IC comparado a crianças ouvintes e outro sobre o desenvolvimento da Escrita por crianças implantadas.

É preciso discutir mais sobre o assunto, divulgar nas escolas e instituições de ensino superior o que é o IC, quais seus benefícios à pessoa implantada e quais as dificuldades mais comuns entre eles, pois como dito no início do artigo o IC não restaura a audição, a criança continua surda, mas há a expectativa que com as sensações auditivas provocadas pelo implante ela desenvolva linguagem oral (TANAMAT; BEVILACQUA; COSTA, 2012). No entanto, o desenvolvimento dessa linguagem depende de diversos fatores, em especial da história de vida criança, na qual se inclui os atendimentos e acompanhamentos condizentes com suas necessidades e dificuldades, e aos processos educativos que vivenciou.

Vale lembrar que dentre os atendimentos previstos pós realização do IC, está o atendimento educacional pedagógico (BRASIL, 1999), ou seja, essa criança tem o direito a um atendimento condizente com suas necessidades, que contribua com seu aprendizado e sucesso escolar, logo estes e outros aspectos relacionados a educação de crianças com IC deveriam ser foco de investigação e pesquisas.

É importante que haja em âmbito nacional uma discussão sobre os benefícios do IC à pessoa surda, em especial àquelas em idade escolar, acompanhando o processo de ensino e aprendizagem vivenciado por essas crianças que estão sendo inseridas na educação regular cada vez em maior número (CAPOVILLA, 1998) e (PINHEIRO et al, 2012). Além desse atendimento/acompanhamento pós cirurgia, garantido por lei, é preciso investir em pesquisas, divulgando o aprendizado vivenciado por elas, as principais dificuldades encontradas tanto no ensino quanto na aprendizagem, o desempenho alcançado, as técnicas e recursos utilizados em sala de aula, e tantos outros temas advindos da realidade educacional dessas crianças.

É importante destacar algumas limitações do estudo: a pesquisa foi realizada em apenas três bases de dados e abrangendo um intervalo curto de investigação (2009-2013), o que pode ter influenciado nos resultados obtidos. 


\section{Conclusões}

A partir da identificação, análise e organização dos artigos internacionais e nacionais sobre educação de pessoas com IC encontrados no período analisado fica evidente o quanto o tema é relevante, e foco de pesquisas em âmbito internacional, e também o déficit de pesquisas nacionais sobre o tema.

A presente pesquisa identificou que as principais tendências em âmbito internacional são estudos descrevendo o processo de aquisição de leitura e educação em geral da pessoa com IC, com foco no trabalho pedagógico realizado com essas pessoas em idade escolar, o que evidencia uma preocupação em complementar o trabalho de reabilitação auditiva pós cirurgia, e o sucesso da pessoa implantada tanto no aspecto auditivo quanto educacional e pessoal.

Em âmbito nacional a presente pesquisa identificou que há muitos estudos sobre IC, em especial estudos desenvolvidos na área da saúde, mas há uma lacuna na área da educação, a qual não tem investido e divulgado o trabalho educacional realizado com pessoas com IC.

Sendo assim, sugere-se maior investimento na área, em especial a temas fundamentais ao processo educacional pós implante, como: Inclusão e educação regular de pessoas com IC, Aquisição de Leitura e Escrita, Recursos e técnicas pedagógicas no ensino e aprendizagem de pessoas com IC, formação e atuação de professores de crianças com IC, dentre tantas outras possibilidades que podem ser identificadas no decorrer da educação dessas pessoas pós cirurgia.

\section{Referências}

BANCO DE DADOS ERIC. Disponível em: eric.ed.gov Acesso em: Jun. 2014.

BANCO DE DADOS LILACS. Disponível em: www.lilacs.bvsalud.org Acesso em: Jun. 2014.

BANCO DE DADOS SCIELO. Disponível em: www.scielo.org Acesso em: Jun. 2014.

BRASIL, PORTARIA No 1.278/MS DE 20 DE OUTUBRO DE 1999. Disponível em: http://bvsms.saude.gov.br/bvs/saudelegis/gm/1999/prt1278_20_10_1999.html Acesso em: Jun. 2014.

CAPOVILLA, F. C. O implante coclear como ferramenta de desenvolvimento linguístico da criança surda. Revista Brasileira Crescimento e Desenvolvimento Humano. São Paulo, 8 (1/2), 1998. 
COSTA FILHO, O.A.; BEVILACQUA, M.C. Implantes Cocleares multicanais em crianças. In: CALDAS NETO, S.C. SIH, T. Otologia e Audiologia em Pediatria. Rio de Janeiro: Revinter, 1999.

DUARTE, J.L.; BAROROTTO, J.S. Análise das estratégias utilizadas em um grupo terapêutico pedagógico para auxiliar o desenvolvimento da linguagem escrita em crianças com deficiência auditiva. Rev. Bras. Ed. Esp, v.15, n.3, 2009 p. 471-484.

IMPLANTE COCLEAR. Disponível em: http:// www.implantecoclear.org.br Acesso em Ago. 2014.

MARCONI, M. A.; LAKATOS, E.M. Técnicas de Pesquisa. São Paulo: Atlas, 1990.

MORET, A. L. M.; BEVILACQUA, M. C.; COSTA, O. A. Implante coclear: audição e linguagem em crianças deficientes auditivas pré-linguais. Pró-Fono Revista de Atualização Científica, Barueri, v. 19, n. 3, 2007, p. 295-304.

PINHEIRO, A.B.S.M.; YAMADA, M.O.; BEVILACQUA, M.C.; CRENITTE, P.A. P. Avaliação das habilidades escolares de crianças com implante coclear. Rev. CEFAC, 2012, p. 826-835.

TANAMATI, L.F; BEVILACQUA, M.C.; COSTA, O.A. Cochlear implant in post lingual children: functional results 10 years after the surgery. Brazilian Journal of Otorrinolaryngology 78 (2), 2012, p. 103-111.

TANAMATI, L.F; COSTA, O.A; BEVILACQUA. M.C. Resultados a longo prazo com uso do implante coclear em crianças: Revisão sistemática. Arq. Int. Otorrinolaringol. V. 15, n.3, p. 365-375,2011.

Recebido em: 01/10/2014

Revisado em: 19/01/2015

Aprovado para publicação em: 30/01/2015

Publicado em: 30/04/2016 